\title{
Suffocating cancer: hypoxia-associated epimutations as targets for cancer therapy
}

\author{
C Thirlwell, LKE Schulz, HK Dibra and S Beck
}

\begin{abstract}
Lower than normal levels of oxygen (hypoxia) is a hallmark of all solid tumours rendering them frequently resistant to both radiotherapy and chemotherapy regimes. Furthermore, tumour hypoxia and activation of the hypoxia inducible factor (HIF) transcriptional pathway is associated with poorer prognosis. Driven by both genetic and epigenetic changes, cancer cells do not only survive but thrive in hypoxic conditions. Detailed knowledge of these changes and their functional consequences is of great clinical utility and is already helping to determine phenotypic plasticity, histological tumour grading and overall prognosis and survival stratification in several cancer types. As epigenetic changes - contrary to genetic changes - are potentially reversible, they may prove to be potent therapeutic targets to add to the cancer physicians' armorarium in the future.

Here, we review the therapeutic potential of epigenetic modifications (including DNA methylation, histone modifications and miRNAs) occurring in hypoxia with particular reference to cancer and tumourigenesis.
\end{abstract}

Keywords: Hypoxia, Hypoxia inducible factor (HIF), DNA methylation, histone modification, micro-RNA

\section{Introduction}

Tumour hypoxia - a regulatory factor in tumour growth Control of cellular oxygen concentration is under strict regulation as hyperoxia causes damage secondary to reactive oxygen species and hypoxia leads to activation of a diverse array of downstream transcriptional pathways including angiogenesis, glucose metabolism and apoptosis.

It is well understood that tumourigenesis is dependent on the development of microvasculature for micronutrient supply and oxygenation. As this vasculature develops in a chaotic way with structural malformations, regions of hypoxia are present within all solid tumours. Normal oxygen tension in healthy tissue is $7 \%(53 \mathrm{mmHg})$, levels of oxygenation in tumours may vary from physiological levels (7\%) to severe hypoxia $(<1 \%)$ which is usually found in areas adjacent to necrotic tissue [1]. It has also been reported that within the same region of a given tumour, levels of oxygenation may cycle due to poor vasculature and limited oxygen diffusion, resulting in intermittent periods of hypoxia [1]. These cyclical episodes of hypoxia lead to increased metastatic potential of cancer cells [2].

\footnotetext{
* Correspondence: christina.thirlwell@ucl.ac.uk

Medical Genomics Laboratory, UCL Cancer Institute, 72, Huntley Street, WC1E $6 \mathrm{BT}$, London
}

(c) 2011 Thirlwell et al; licensee BioMed Central Ltd. This is an Open Access article distributed under the terms of the Creative Commons Attribution License (http://creativecommons.org/licenses/by/2.0), which permits unrestricted use, distribution, and reproduction in any medium, provided the original work is properly cited. Cellular hypoxia is toxic, and when severe leads to cell
death in both normal and cancerous cells. However, cancer cells have developed mechanisms through (epi)genetic modifications which allow them to survive and in some cases thrive in hypoxic conditions [3]. Preclinical studies have demonstrated that the selective pressure of tissue hypoxia drives the selective outgrowth of more aggressive tumour sub-clones [4]. In early stage lung cancer and pancreatic endocrine cancer markers of hypoxia have outperformed traditional histopathological staging for predicting prognosis $[5,6]$.

The direct observation of tumour hypoxia through oxygen electrode recording was first made over ten years ago [7]. This study demonstrated the relationship between low oxygen tension and increased risk of metastasis along with poor prognosis in head and neck, breast and cervical cancers. It has since been possible to demonstrate hypoxia in vivo through the injection of pimidiazole [8].

The hypoxia inducible factor (HIF) pathway is the key regulatory pathway activated in response to tissue hypoxia. In normoxic conditions HIF is degraded through interaction with the Von-Hippel Lindau (VHL) tumour suppressor protein ( $\mathrm{pVHL}$ ) where it becomes polyubiquinated and undergoes proteosomal degradation. The interaction between VHL and HIF is controlled via 
post-translational prolyl hydroxylation of HIF through prolyl hydroxylases (PHDs). In hypoxic conditions HIF does not bind to VHL due to the action of PHDs and therefore accumulates. HIF-1 $\alpha$ then binds to hypoxia response elements (HRE) along with co-factors HIF-1 $\beta$ (also known as arylhydrocarbon receptor nuclear factor (ARNT)), E1A binding protein p300 (EP300), jun protooncogene (c-JUN) and cAMP responsive element binding protein (CREB). This leads to the transcription and upregulation of over 100 genes involved in angiogenesis, glucose metabolism and transportation, erythropoietin production, cellular proliferation, tumour invasion/ metastasis and $p 53$ mediated apoptosis $[9,10]$. Three HIF- $\alpha$ subunits have been identified, HIF- $1 \alpha$ and HIF- $2 \alpha$ are thought to have roles in acute and chronic hypoxia respectively whereas the role of HIF-3 $\alpha$ is yet to be determined [11]. In vitro studies in several cell culture systems have shown that HIF is activated at approximately 5\% $\mathrm{O}_{2}$ $(40 \mathrm{mmHg})$ and activity increases with decreasing oxygenation down to $0.2-1.0 \%(1.6-0.8 \mathrm{mmHg}) \mathrm{O}_{2}$ nearing anoxia [12]. See Figure 1 for an overview of the HIF pathway (adapted from Biocarta, http://www.biocarta.com). As HIF activation leads to the development of tumour vascularisation, it plays a significant role in tumour progression and metastasis. Over-expression of HIF-1 $\alpha$ and HIF- $2 \alpha$ has been documented in several primary tumours and associated metastases, the degree of expression correlates with angiogenesis, resistance to treatment and overall patient outcome $[9,3]$. The HIF pathway can also be activated through germline or somatic mutation of $V H L$ or through $V H L$ promoter methylation leading to transcriptional repression. Renal cell carcinoma can develop through all of these mechanisms (reviewed in $[13,14])$. For a review of the role of HIF in tumourigenesis see $[12,3,15]$. As HIF activation can be deleterious to both cancerous and normal cells, feedback mechanisms exist to control the level of HIF activation, which leads to a switch from HIF- $1 \alpha$ to HIF- $2 \alpha$ driven response when acute hypoxia becomes chronic. HIF- $2 \alpha$ driven responses occurring in chronic hypoxia may be involved in the regulation of tumour cellular differentiation and stem cell maintenance (reviewed in [16]).

Alongside HIF related transcriptional response to tissue hypoxia, there is increasing evidence to support the development of hypoxia mediated epigenetic modifications which lead to further transcriptional changes and chromosomal instability. This is mediated through changes in DNA methylation at promoter regions and satellite repeats [17], histone modifications [18], and micro-RNAs [19]. Further genetic instability in hypoxia is due to gene amplification and DNA strand breaks at fragile sites alongside disruption of DNA repair [20].

Phenotypic transition can also occur as a result of hypoxia, with evidence of epithelial - mesenchymal transition (EMT) [21] and the development of tumour specific cancer stem cells (CSC), as seen in glioblastoma [1] and breast cancer [22]. CSCs are phenotypically similar to normal stem cells and behave similarly in that they are able to up-regulate DNA repair enzymes [23].

\section{Epigenetic manifestations of hypoxia in normal tissue}

It is clear that the physiological response to hypoxia in normal tissues and organs must involve epigenetic modifications which lead to downstream changes in gene expression which have either protective or adaptive functions. The following studies have found evidence of a global increase in DNA methylation following a period of hypoxia with associated changes in transcriptional activation and repressive histone marks.

RNA expression array studies on murine placenta identified increased expression of DNA methyltransferase 3B (Dnmt3b), and methyl-CpG binding domain protein 1 (Mbd1) following a 48 hour period of hypoxia [24]. An increase in the expression of genes involved in metabolism, oxygen transport, proteolysis, cell death and reactive oxygen species metabolism was also identified. It was concluded from this study that hypoxia may contribute to long-term epigenetic changes in hypoxically stressed tissues and organs. Previous studies have demonstrated that reactive oxygen species can affect DNA methylation [25] suggesting a mechanism for the increased expression of DNA methyltransferases in hypoxia.

Increased expression of DNA methyltransferases during hypoxia in human tissues was confirmed when Watson et al determined the epigenetic signature of chronic hypoxia in normal prostate cell lines [26]. Following a 7 week period of normoxic culture, cells were then cultured for a further 4 weeks at $3 \% \mathrm{O}_{2}$ and then 3 weeks at $1 \% \mathrm{O}_{2}$ prior to assessment of global DNA methylation, DNMT and histone deacetylase activity. This found a global increase in DNA methylation with concomitant increase in DNMT3B expression and H3K9 histone acetylation. Gene-specific changes in DNA methylation were also observed at several key loci of imprinted genes.

Hypoxia is known to induce alterations in chromatin, including global deacetylation and also changes in histone methylation and acetylation in the promoter regions of hypoxia related genes. Chromatin precipitation analysis has identified a signature of chromatin modifications which is induced

under hypoxic stress. It has been suggested that this may play a role in gene regulation in proliferating tumour cells undergoing cyclic periods of hypoxia [27].

The jumonji-domain containing dioxygenases (JMJD) group of histone demethylases (JHDMs) have been recently described and are found to be regulated in hypoxic conditions through HIF-1 $\alpha$ [28]. Within the JMJD group, JMJD1A and JMJD2B were found to be 


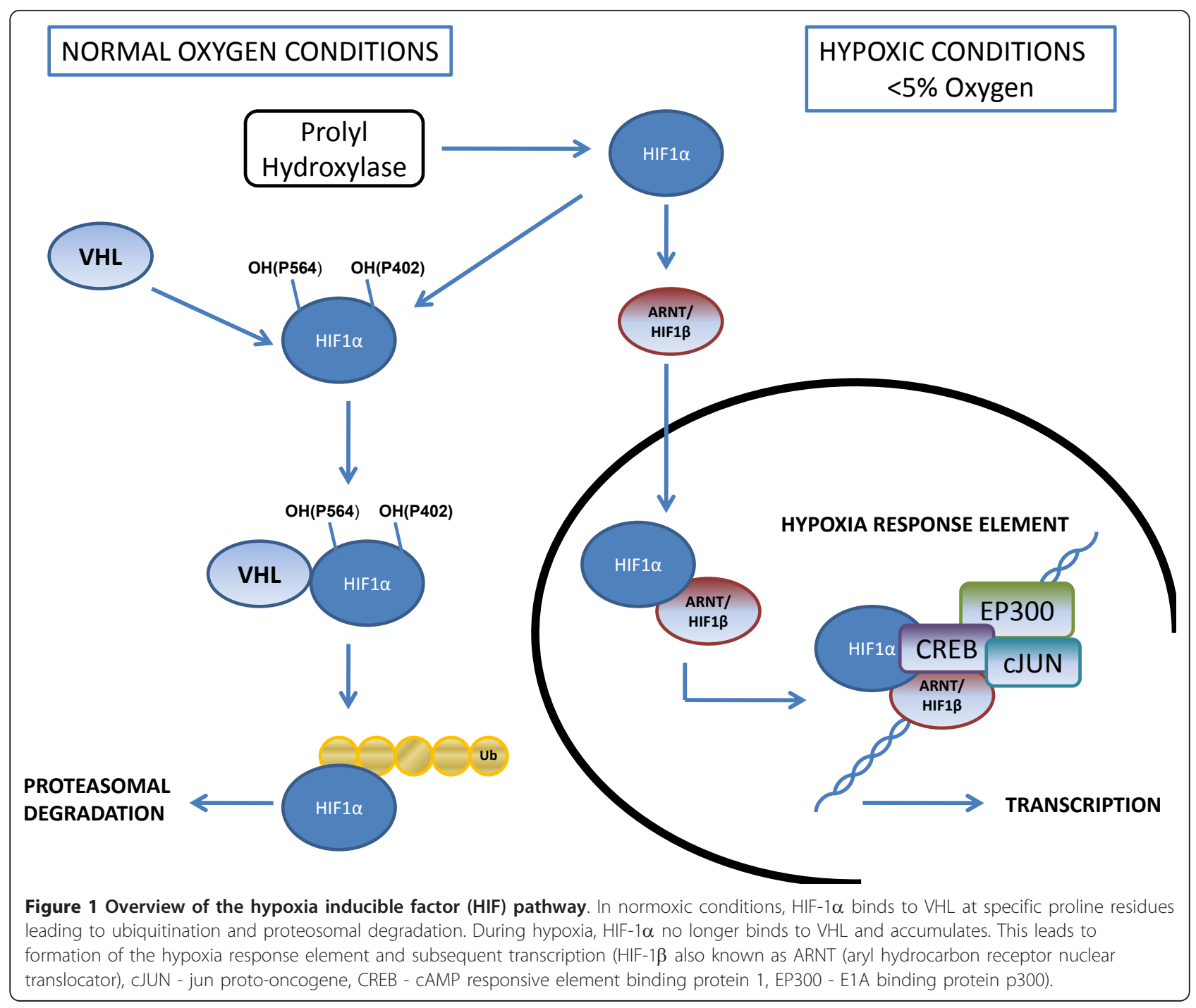

significantly up-regulated during hypoxia with JMJD2C showing modest up-regulation. This hypoxia related dynamic control of histone methylation is thought to regulate chromatin assembly and gene expression. JMJDs affect gene expression through demethylation of lysine residues of histone tails. In particular, JMJD1A has been found to demethylate $\mathrm{H} 3 \mathrm{~K} 9 \mathrm{me} 1$ and $\mathrm{H} 3 \mathrm{~K} 9 \mathrm{me} 2$ and JMJD2B demethylates H3K9me3. Transcriptional pathways have also been associated with individual JMJDs, JMJD1A is associated with the androgen receptor (AR) and regulates AR target genes. Both JMJD1A and JMJD2C regulate and increase the expression of genes involved in self-renewal and are targets for the transcription factor Oct- 4 in embryonic stem cells[28].

Although all of these studies have documented a global increase in DNA methylation and histone modifications, which are associated with changes in downstream transcription, there are no studies to date which document the time course or order of these events. It is very likely that different cell and tissue types would require varying periods of hypoxia before "normal" hypoxic cells become "precancerous" or "cancerous" hypoxic cells.

\section{Epigenetic manifestations of hypoxia in tumour development \\ DNA methylation}

The observation of global hypomethylation was first observed in colorectal cancer cell lines in the 1980's [29]. Over the following twenty years a number of tumour suppressor genes were found to be silenced through promoter region hypermethylation [30]. The first DNA methylome (at single nucleotide resolution) was published in 2008, this was followed in quick succession by several other studies which have given us a wealth of information regarding the function of DNA methylation in both coding and non-coding regions of the genome 
[31]. For example, the concept of $\mathrm{CpG}$ island shores was developed (regions of lower CpG density occurring within $2 \mathrm{~kb}$ of CpG islands) [32]. These regions have since been found to harbour the majority of tissue specific differentially methylated regions of DNA [33].

There are few studies which have investigated genomewide DNA methylation changes occuring during hypoxia. Methylation within DNA repeat elements (retrotransposable $A l u$ and other short interspersed nuclear elements (SINEs)) is known to contribute to genomic stability, it has been postulated that chronic hypoxia can lead to global hypomethylation and subsequent increase in genomic instability and aneuploidy which is often observed in tumourigenesis. This is supported by two studies $[17,34]$ reporting an increase in global hypomethylation in glioblastoma, sarcoma, colorectal cancer and melanoma cell lines when cultured in hypoxic conditions when compared to normoxic culture. The study of glioblastoma and sarcoma cell lines used RT-PCR methodology alongside bisulphite sequencing of $A l u$ and other SINE repeat regions within the genome, whereas the study of colorectal cancer and melanoma cell lines used HPLC to quantify 5 -methylcytosine $(5-\mathrm{mC})$. These findings are the exact opposite of those observed when culturing noncancerous cells in hypoxic conditions (ie an increase in global methylation) and may in part be due to a breakdown in the feedback mechanisms which exist to control the degree of HIF activation occurring in cancer cells and not normal cells during hypoxia.

\section{Histone modifications}

The most common histone modifications occurring in cancer are acetylation, deacetylation or methylation at lysine residues. These modifications are catalysed by histone deacetylases (HDACs) and histone methyltranferases (HMTs) [35]. Histone acetylation is most commonly associated with repression of transcription, whereas histone methylation is associated with both transcriptional activation and repression. A number of methylation reactions are associated with transcriptional activity (di- and trimethylation of H3K4 (H3K4me2 and H3K4me3), trimethylation of H3K36 (H3K36me3) and dimethylation of H3K79 (H3K79me2). In contrast, di- and trimethylation at H3K9, H3K27 and $\mathrm{H} 4 \mathrm{~K} 20$ is associated with gene silencing [36,37]. Specifically in cancer, a global loss of H4K16ac is seen alongside loss of the transcriptionally active mark H3K4me3 and gain of the transcriptionally repressive marks H3K9me and H3K27me [38].

Histone modifying enzymes play a key role in hypoxiarelated tumourigenesis It is increasingly evident that histone modifying enzymes play a key role in hypoxiarelated tumourigenesis. Several research groups have demonstrated that hypoxia regulates the activity of histone demethylases [18]. Xia et al used ChIP-chip and RNA expression profiling of HepG2 (hepatocellular cancer cell line) and U87 (glioma cell line) cells grown in normoxic (ambient) and hypoxic $\left(0.5 \% \mathrm{O}_{2}\right.$ for 4 hours) conditions to define HIF-1 chromatin binding targets. In total, 377 HIF-1 binding sites activated by hypoxia were identified across the genome. As predicted, many of the genes associated with these binding sites were from biological pathways known to be activated and controlled by HIF. However, enrichment for the 2-oxoglutarate dioxygenase family of enzymes was also observed including the previously described JHDMs. It was further demonstrated that up-regulation of these JHDMs helps to maintain histone homeostasis during hypoxic tumorigenesis [18]. The JHDM family of histone demethylases do not only require oxygen to function but are in some cases induced during hypoxia through HIF related mechanisms [11]. The JHDMs, JMJD2B and JMJD2C have been found to have oncogene-like propertied and are amplified in squamous cell of the oesophagus and medulloblastoma [39,40].

In addition, inactivating mutations in enzymes controlling histone modification have been detected in clear cell renal carcinoma (ccRCC), clear cell ovarian cancer [41] and pancreatic neuroendocrine tumours [42]. The histone modifying enzymes directly involved in ccRCC development are SET domain containing 2 (SETD2), a histone H3 lysine 36 methyltransferase, Jumonji/ARID domain-containing protein $1 \mathrm{C}$ (JARID1C), a histone $\mathrm{H} 3$ lysine 4 demethylase and ubiquitously transcribed $\mathrm{X}$ chromosome tetratricopeptide repeat protein (UTX) a histone H3 lysine 27 demethylase $[43,44]$. Even though a small fraction of clear cell renal cell carcinoma (3\%) cases harboured inactivating mutations in SETD2 or JARID1C, these mutations are potentially of high relevance in hypoxia since $88 \%$ of samples with SETD2 and JARID1C inactivating mutations also harboured mutations in $V H L$ and/or over-expression of egl nine homolog 3 ( $E L G N 3$ ) which is also known as HIF-prolyl hydroxylase 3 (PHD3) [43]. JARID1B, a histone $\mathrm{H} 3$ lysine 4 demethylase is found to be up-regulated in prostate cancer [45].

In gastric cancer, a hypoxia-induced epigenetic silencing of the tumour suppressor RUNX3 through histone H3-lysine 9 dimethylation and decreased $\mathrm{H} 3$ acetylation during disease progression has been observed [46]. RUNX3 is also known to be silenced in several cancers through promoter methylation. This study performed on gastric cancer cell lines revealed that following treatment with the histone deacetylase inhibitor trichostatin A and the cytosine-methylation inhibitor 5-aza-2-deoxycytidine, down-regulation of RUNX3 was reversed. Bisulphite-specific PCR demonstrated that promoter region methylation was unchanged in hypoxia, whereas chromatin immunoprecipitation (CHIP) found an increase in the repressive histone mark $\mathrm{H} 3 \mathrm{~K} 9 \mathrm{me} 2$ and a decrease in the transcriptionally active H3K9 acetylation mark. It was also found that the G9a histone methyltransferase and 
HDAC1 were up-regulated following hypoxia, alongside diminished nuclear localisation and expression of $R U N X 3$, demonstrating an epigenetic mechanism for the observed decrease in RUNX3 expression.

Hypoxic induction of histone demethylases therefore play a pivotal role in tumourigenesis, one such mechanism being HIF- $\alpha$ mediated induction of JHDMs leading to cancer progression. The role of histone methyltransferases which may oppose the effects of histone demethylases during hypoxia is less well understood. If the effects of these two opposing families of histone modifying enzymes during hypoxia are determined, a vital connection between environmental stressors and epigentically regulated tumourigenesis would be made and furthermore, novel epigenetic cancer therapeutic targets identified.

\section{Micro-RNAs}

Micro-RNAs (miRNAs) are short (20-24) non-coding nucleotide RNA molecules [47]. They are thought to regulate gene expression through control of mRNA turnover, inhibition of translation, promoter region activation and epigenetic silencing [48]. The "micro-transcriptome" is thought to account for 1-2\% of the human genome and regulate the majority of translated genes [49]. miRNAs have the ability to "respond" both immediately and reversibly to hypoxic stress. miRNAs identified and implicated in tumorigenesis when normal tissue and tumour samples are compared [50] are affected through several factors in the tumour microenvironment such as hypoxia, $\mathrm{pH}$, glucose metabolism and paracrine growth factors. Hypoxia regulated miRNAs are implicated in both renal cell cancer an glioblastoma development, several other hypoxia regulated miRNAs are in development and cancer bioamarkers, these are discussed below.

Hypoxia related mi-RNAs can be used as diagnostic and prognostic cancer biomarkers Kulshreshtha et al have identified a group of hypoxia regulated miRNAs (HRMs). Several of these miRNAs regulate apoptotic signalling during hypoxic periods. The majority of the identified HRMs were found to be over-expressed in human cancers and were consistently induced in breast and colorectal cancer cell lines during culture in hypoxic conditions $[51,52]$. Other groups have also reported induction of miRNAs as a response to hypoxia [53] but it is difficult to compare studies directly due to variation in culture times and degree of hypoxia used for culturing between research groups.

miR-210 is known to be regulated by hypoxia and has found to be a diagnostic marker in pancreatic adenocarcinoma [54] and prognostic marker in breast cancer [55]. miR-210 is also over-expressed in late stage non-small cell lung cancer [56]. Subsequent in vitro studies on the lung adenocarcinoma cell line A549 demonstrated that miR210 expression results in caspase 3/7 activity, followed by mitochondrial dysfunction. In the same study, subunit D of succinate dehydrogenase complex (SDHD) was validated as a miR-210 target [56].

The relationship between the HIF pathway and hypoxia-mediated miRNAs Neal et al first demonstrated the impact of the $V H L$ tumour suppressor gene on miRNAs in renal clear cell carcinoma [57]. This study elegantly showed that miRNAs are downstream effectors of the HIF-induced hypoxia response. Under hypoxic conditions (or through $V H L$ mutation or $V H L$ promoter methylation), miR-210 expression is significantly increased and potentially targets the iron-sulphur cluster protein (ISCU). ISCU is implicated in the mitochondrial electron transport chain and potentially, in anaerobic respiration of tumours. A further group of miRNAs has been identified which regulate VEGF (miR-16, 20a, 20b, 17-5p, 27a, 106a, 106b, 107, 193a, 210, 320, 321) [58].

The over expression of miR-21 (a HRM) has been implicated in the development of glioblastoma (which is known to have extensive regions of hypoxia and necrosis), and knock-down of this miRNA in glioblastoma cell lines lead to apoptosis, suggesting a role in tumour survival [59].

Although none of the hypoxia regulated miRNAs described above are used routinely for clinical diagnosis and prognosis to date, these biomarkers once validated, are easily accessible from both tissue biopsies, resections and are found in circulating plasma enabling clinical access for both diagnostic and prognostic purposes.

\section{Potential epigenetic targets for hypoxia related cancer therapy \\ DNA methyltransferase inhibitors}

DNA methyltransferase (DMNT) inhibitors have been investigated for some years as a novel class of anti-cancer drug. There is substantial evidence to support the use of the DNMT inhibitors 5-azacytidine and 5-aza-2'-deoxycytidine (decitabine) for certain haematological malignancies, resulting in their approval by the FDA. Following a number of phase I and II trials the evidence for their use in solid tumours is less encouraging. In light of this, these agents have not been trialled with specific reference to tumour hypoxia. Treatment with decitabine has been shown to lead to re-expression of $V H L$ in clear cell renal carcinoma in vitro and in vivo through inhibition of DNA methylation in the $V H L$ promoter region [60]. This leads to restoration of the normal physiological HIF pathway and reduction of accumulation of HIF- $1 \alpha$ and downstream hypoxia response element related transcription. Re-expression of BNIP3 which is associated with hypoxia mediated apoptosis following treatment with decitabine has also been observed in pancreatic adenocarcioma cell lines [61].

\section{Histone Deacetylase Inhibitors}

HDAC inhibitors are compounds which function through inhibition of deacetylases, they are a diverse group of 
compounds which are divided in to classes I-IV. HDAC inhibitors do not act solely as inhibitors of HDACs as a large number of non-histone transcription factors and transcriptional co-regulators are known to be modified by acetylation.

A clear mechanism of action of these drugs is yet to be elucidated. To date, they have been found to have proapoptopic and anti-angiogenic properties. Several HDAC inhibitors are currently under evaluation in clinical trials both a single agents and in combination with chemotherapry and targeted therapy [62-64]. The second generation HDAC inhibitor Vorinostat has been approved by the FDA for the treatment of cutaneous $T$ cell lymphoma.

Recently it has been shown that the class I and II HDAC inhibitors disrupt HIF both directly through reduction of HIF-1 $\alpha$ levels and indirectly through repressing transcription, the exact mechanism of this remains unknown [65]. A number of HDAC inhibitors have been reported to down-regulate HIF-1 $\alpha$ stability in cancer cell lines. It has been suggested that the transcription complexes of HIF-1 $\alpha$ and HIF- $2 \alpha$ require modification by type I/II deacetylases in order to become transcriptionally active, it is through this mechanism that HDAC inhibitors are thought to reduce HIF transcription [66]. Fath

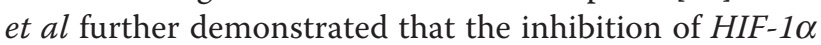
and HIF- $2 \alpha$ transactivation mediated by HDAC inhibitors was independent of VHL and p53 function. This is a significant finding as it implies that HDAC inhibitors may have a therapeutic effect in VHL deficient tumours (such a renal cell cancer and pancreatic neuroendocrine tumours). The class III HDAC inhibitor SirtI has also been found to deacetylate HIF-1 $\alpha$ and HIF- $2 \alpha$ thus repressing HIF activity [67].

Sodium butyrate, a novel HDAC inhibitor has been shown to inhibit hypoxia induced $H I F 1 \alpha$ induction and inhibited in vitro and in vivo angiogenesis [68]. The study demonstrated that sodium buyrate treatment of endothelial cells down-regulated both HIF1 $\alpha$ and VEGF protein levels thereby regulating angiogenesis. This indicates that HDACs are involved in oxygen dependant gene expression and angiogenesis.

Combination of HDAC inhibitors with other targeted treatments can have an additive effect Verheul et al., demonstrated in prostate and renal cancer cell lines that combination treatment with the mTOR inhibitor rapamycin and the HDAC inhibitor LBH589, significantly reduced HIF- $1 \alpha$ protein expression and consequently suppressed tumour induced angiogenesis on comparison with either agent used singularly [69].

The effects of HDAC inhibitors on both HIF degradation and transcription are therapeutically very appealing and further clinical evaluation should be considered trialing other targeted treatments such as mTOR and VEGF inhibitors alongside HDAC inhibition. It should be noted however that long term systemic administration of HDAC inhibitors may lead to downstream effects on erythropoeisis and ischaemia (including coronary ischaemia) through inhibition of the physiological role that HIF plays in erythoropoietin production, and adaptive metabolic changes that occur during hypoxia. Therefore scheduling and timing of these therapies requires robust phase I trial assessment.

\section{mi-RNA targeted therapy}

Through the identification of specific miRNAs involved in tumorigenesis (in both normoxic and hypoxic conditions) it is deemed feasible to inhibit specific miRNAs in the future through anti-miRNA oligonucleotides [70], locked nucleic acid-modified oligonucleotides [71] and antagomirs [72]. To date, none of these agents have progressed further than phase I clinical trials.

There is recent evidence supporting the potential use of anti-oxidants and naturally occurring compounds to regulate miRNA expression in cancer cells [73]. Li et al [74] demonstrated down-regulation of miR-135a and miRNA $135 \mathrm{~b}$ in colorectal cancer cell lines (which target the 3' untranslated region of adenomatous polyposis coli gene) through treatment with mistletoe lectin-I, the mechanism of this is thought to be through degradation of the miRNA precursors. Anti-oxidants such as Se-Methylselenocysteine (MSC) and N-acetyl cysteine (NAC) have been shown to inhibit HIF- $1 \alpha$ in solid tumours in mouse models [75,76], historically it was thought that anti-oxidants had an antitumourigenic effect through the reduction of reactive oxygen species (ROS) and subsequent DNA damage, these studies demonstrate a direct inhibition of HIF by anit-oxidants. MSC when administered alongside irinotecan chemotherapy improved clinical outcome in mice with human squamous cell tumour xenografts through inhibition of HIF-1 $\alpha$ [75] and NAC was shown to reduce HIF activity in mouse models of hepatocellular carcinoma and B cell lymphoma [76].

The role of miR-210 in relation to tumour hypoxia has been recently reviewed [77]. Alongside its potential use as a novel hypoxia diagnostic marker in pancreatic cancer $[54,78]$ and prognostic biomarker in breast [55], head and neck [79] and renal cancer [57]. Although these studies do not demonstrate a direct mi-RNA targeted therapy, they illustrate a novel potential of these epigenetic markers within a clinical setting to help aid cancer diagnosis via non-invasive methods and ultimately impact on patient treatment and prognosis. Table 1 gives an overview of tumourigenic hypoxia related epigenetic changes and potential therapeutic strategies and targets.

\section{Conclusions and outlook}

It is clear that epigenetic changes occurring during hypoxia have a significant downstream effect physiologically in normal tissue, in the transition from normal to 
Table 1 Tumourigenic hypoxia related epigenetic changes and potential therapeutic strategies and targets.

\begin{tabular}{|c|c|c|}
\hline & $\begin{array}{c}\text { Modification observed in hypoxia related } \\
\text { tumourigenesis }\end{array}$ & Therapeutic potential \\
\hline DNA methylation & Global DNA hypomethylation & $\begin{array}{l}\text { • DNMT inhibition in haematological malignancies } \\
\text { - Re-expression of specific tumour suppressor genes (eg. VHL) through } \\
\text { decitabine therapy }\end{array}$ \\
\hline $\begin{array}{l}\text { Histone } \\
\text { modification }\end{array}$ & $\begin{array}{c}\text { - Hypoxic regulation of histone demethylase } \\
\text { activity } \\
\text { - Up-regulation of HDAC1 and histone } \\
\text { methyltransferase }\end{array}$ & $\begin{array}{c}\cdot \text { HDAC inhibitors disrupt HIF through altered transcription and } \\
\text { degradation } \\
\text { - Combination of HDAC inhibiton with mTOR inhibition has additive effect } \\
\text { in vitro }\end{array}$ \\
\hline miRNAs & $\begin{array}{l}\text { mi-RNAs associated with diagnosis and prognosis } \\
\qquad \text { eg mi-210 }\end{array}$ & $\begin{array}{c}\text { - Selected hypoxia related } \\
\text { mi-RNA inhibition through } \\
\text { - Anti-miRNA oligonucleotides } \\
- \text { Antagomirs } \\
\text { - Indirect regulation of miRNA through HIF inhibitors }\end{array}$ \\
\hline
\end{tabular}

cancerous tissue and in the progression of solid tumours. These changes can be used as biomarkers in diagnosis and prognosis, for example the micro-RNA miR-210 in pancreatic and breast cancer.

However, in order to fully understand these changes and harness their clinical benefit for diagnostics and therapeutics, several key areas need to be addressed which are discussed below.

In normal tissue global DNA hypermethylation is observed following a period of hypoxia whereas in cancer cell lines global DNA hypomethylation is observed leading to increased genomic instability. This is likely due to decreased DNA methyltransferase activity - both maintenance and de novo. What we do not know is whether the global DNA hypomethylation observed in hypoxic cancer tissue is somehow protective enabling survival in hypoxic conditions.

It is not known whether a stepwise chain of epigenetic events occurs during hypoxia, ie changes in DNA methylation occurring before histone modifications and alteration in expression of downstream mi-RNAs. If this sequence of events could be determined it might highlight the best diagnostic and therapeutic strategies to employ in different cancer types. In reality it is likely that there is interaction and positive and negative feedback between all epigenetic modifications.

Therapeutically, DNMT inhibitors have not been trialled with respect to tumour hypoxia. However HDAC inhibitors appear to have a direct effect on both HIF transcription and degradation making them promising therapeutic agents for use in vascular tumours and tumours with severe regions of hypoxia. If used in the right clinical setting for selected tumour types this therapeutic intervention could prove to be clinically beneficial. Future therapeutic strategies could include the integration of HDAC inhibition with other targeted therapies such mTOR and VEGF/c-kit/platelet derived growth factor (PDGF) tyrosine kinase inhibitors. Sequencing and duration of treatment with such therapies in combination is yet to be determined.

With the recent massive expansion of molecular biology tools available for studying the epigenome (both array and sequencing based) [80], we are at a turning point in furthering our understanding of the effects of hypoxia on the epigenome and identifying novel clinical biomarkers and therapeutic targets.

\section{Acknowledgements}

$\mathrm{CT}$ and HKD are funded by Cancer Research UK and the Raymond and Beverly Sackler Foundation.

SB is funded by the Wellcome Trust.

We would like to thank AF for helpful comments and suggestions during preparation of the manuscript.

\section{Authors' contributions}

$C T$ drafted and revised the manuscript, LKES contributed to content relating to miRNAs and HKD contributed to content relating to histone

modifications. SB provided guidance for the overall structure and content of the manuscript. All authours read and approved the final manuscript.

\section{Competing interests statement}

The authors declare that they have no competing interests.

Received: 16 August 2011 Accepted: 5 December 2011

Published: 5 December 2011

\section{References}

1. Heddleston JM, et al: Hypoxia inducible factors in cancer stem cells. $\mathrm{Br}$ J Cancer 2010, 102(5):789-95.

2. Cairns RA, Kalliomaki T, Hill RP: Acute (cyclic) hypoxia enhances spontaneous metastasis of KHT murine tumors. Cancer Res 2001, 61(24):8903-8.

3. Harris AL: Hypoxia-a key regulatory factor in tumour growth. Nat Rev Cancer 2002, 2(1):38-47.

4. Anderson AR, et al: Tumor morphology and phenotypic evolution driven by selective pressure from the microenvironment. Cell 2006, 127(5):905-15.

5. Kim SJ, et al: Carbonic anhydrase IX in early-stage non-small cell lung cancer. Clin Cancer Res 2004, 10(23):7925-33.

6. Couvelard A, et al: Microvascular density and hypoxia-inducible factor pathway in pancreatic endocrine tumours: negative correlation of microvascular density and VEGF expression with tumour progression. $\mathrm{Br}$ J Cancer 2005, 92(1):94-101.

7. Hockel M, Vaupel P: Tumor hypoxia: definitions and current clinical, biologic, and molecular aspects. J Natl Cancer Inst 2001, 93(4):266-76. 
8. Varia MA, et al: Pimonidazole: a novel hypoxia marker for complementary study of tumor hypoxia and cell proliferation in cervical carcinoma. Gynecol Oncol 1998, 71(2):270-7.

9. Semenza GL: Targeting HIF-1 for cancer therapy. Nat Rev Cancer 2003, 3(10):721-32.

10. Suzuki H, Tomida A, Tsuruo T: Dephosphorylated hypoxia-inducible factor 1 alpha as a mediator of p53-dependent apoptosis during hypoxia. Oncogene 2001, 20(41):5779-88.

11. Yang J, et al: Role of hypoxia-inducible factors in epigenetic regulation via histone demethylases. Ann N Y Acad Sci 2009, 1177:185-97.

12. Pouyssegur J, Dayan F, Mazure NM: Hypoxia signalling in cancer and approaches to enforce tumour regression. Nature 2006, 441(7092):437-43.

13. Kim WY, Kaelin WG: Role of VHL gene mutation in human cancer. $J$ Clin Oncol 2004, 22(24):4991-5004.

14. Gossage L, Eisen T: Alterations in VHL as potential biomarkers in renalcell carcinoma. Nat Rev Clin Oncol 2010, 7(5):277-88.

15. Maxwell PH: The HIF pathway in cancer. Semin Cell Dev Biol 2005, 16(45):523-30.

16. Henze AT, Acker T: Feedback regulators of hypoxia-inducible factors and their role in cancer biology. Cell Cycle 2010, 9(14):2749-63.

17. Pal A, et al: Aberrant methylation and associated transcriptional mobilization of Alu elements contributes to genomic instability in hypoxia. J Cell Mol Med 2010, 14(11):2646-54.

18. Xia X, et al: Integrative analysis of HIF binding and transactivation reveals its role in maintaining histone methylation homeostasis. Proc Natl Acad Sci USA 2009, 106(11):4260-5.

19. Cortez MA, et al: microRNAs in cancer: from bench to bedside. Adv Cancer Res 2010, 108:113-57.

20. Coquelle $A$, et al: A new role for hypoxia in tumor progression: induction of fragile site triggering genomic rearrangements and formation of complex DMs and HSRs. Mol Cell 1998, 2(2):259-65.

21. Polyak K, Weinberg RA: Transitions between epithelial and mesenchymal states: acquisition of malignant and stem cell traits. Nat Rev Cancer 2009, 9(4):265-73.

22. Louie $E$, et al: Identification of a stem-like cell population by exposing metastatic breast cancer cell lines to repetitive cycles of hypoxia and reoxygenation. Breast Cancer Res 2010, 12(6):R94.

23. Bao $S$, et al: Stem cell-like glioma cells promote tumor angiogenesis through vascular endothelial growth factor. Cancer Res 2006, 66(16):7843-8.

24. Gheorghe $C P$, et al: Gene expression patterns in the hypoxic murine placenta: a role in epigenesis? Reprod Sci 2007, 14(3):223-33.

25. Fratelli $M$, et al: Gene expression profiling reveals a signaling role of glutathione in redox regulation. Proc Natl Acad Sci USA 2005, 102(39):13998-4003.

26. Watson JA, et al: Generation of an epigenetic signature by chronic hypoxia in prostate cells. Hum Mol Genet 2009, 18(19):3594-604

27. Johnson $A B$, Denko $N$, Barton MC: Hypoxia induces a novel signature of chromatin modifications and global repression of transcription. Mutat Res 2008, 640(1-2):174-9.

28. Pollard PJ, et al: Regulation of Jumonji-domain-containing histone demethylases by hypoxia-inducible factor (HIF)-1alpha. Biochem J 2008, 416(3):387-94.

29. Feinberg AP, Vogelstein B: Hypomethylation distinguishes genes of some human cancers from their normal counterparts. Nature 1983, 301(5895):89-92.

30. Jones PA, Baylin SB: The fundamental role of epigenetic events in cancer. Nat Rev Genet 2002, 3(6):415-28.

31. Lister $R$, et al: Human DNA methylomes at base resolution show widespread epigenomic differences. Nature 2009, 462(7271):315-22.

32. Irizarry RA, et al: The human colon cancer methylome shows similar hypo- and hypermethylation at conserved tissue-specific CpG island shores. Nat Genet 2009, 41(2):178-86.

33. Doi A, et al: Differential methylation of tissue- and cancer-specific CpG island shores distinguishes human induced pluripotent stem cells, embryonic stem cells and fibroblasts. Nat Genet 2009, 41(12):1350-3.

34. Shahrzad $S$, et al: Induction of DNA hypomethylation by tumor hypoxia. Epigenetics 2007, 2(2):119-25.

35. Johnstone RW: Histone-deacetylase inhibitors: novel drugs for the treatment of cancer. Nat Rev Drug Discov 2002, 1(4):287-99.
36. Berger SL: The complex language of chromatin regulation during transcription. Nature 2007, 447(7143):407-12.

37. Kouzarides T: Chromatin modifications and their function. Cell 2007, 128(4):693-705.

38. Portela A, Esteller M: Epigenetic modifications and human disease. Nat Biotechnol 2010, 28(10):1057-68.

39. Yang ZQ, et al: A novel amplicon at $9 p 23-24$ in squamous cell carcinoma of the esophagus that lies proximal to GASC1 and harbors NFIB. Japanese journal of cancer research: Gann 2001, 92(4):423-8.

40. Northcott PA, et al: Multiple recurrent genetic events converge on control of histone lysine methylation in medulloblastoma. Nature genetics 2009, 41(4):465-72

41. Jones $S$, et al: Frequent mutations of chromatin remodeling gene ARID1A in ovarian clear cell carcinoma. Science 2010, 330(6001):228-31.

42. Jiao $Y$, et al: DAXX/ATRX, MEN1, and mTOR pathway genes are frequently altered in pancreatic neuroendocrine tumors. Science 2011, 331(6021):1199-203.

43. Dalgliesh $\mathrm{GL}$, et al: Systematic sequencing of renal carcinoma reveals inactivation of histone modifying genes. Nature 2010, 463(7279):360-3.

44. van Haaften $\mathrm{G}$, et al: Somatic mutations of the histone H3K27 demethylase gene UTX in human cancer. Nat Genet 2009, 41(5):521-3.

45. Xiang $Y$, et al: JARID1B is a histone $\mathrm{H} 3$ lysine 4 demethylase up-regulated in prostate cancer. Proceedings of the National Academy of Sciences of the United States of America 2007, 104(49):19226-31.

46. Lee $\mathrm{SH}$, et al: Hypoxic silencing of tumor suppressor RUNX3 by histone modification in gastric cancer cells. Oncogene 2009, 28(2):184-94

47. Ambros V: microRNAs: tiny regulators with great potential. Cell 2001, 107(7):823-6.

48. Calin GA, Croce CM: MicroRNA signatures in human cancers. Nat Rev Cancer 2006, 6(11):857-66.

49. Kloosterman WP, Plasterk RH: The diverse functions of microRNAs in animal development and disease. Dev Cell 2006, 11(4):441-50.

50. Volinia $S$, et al: A microRNA expression signature of human solid tumors defines cancer gene targets. Proc Natl Acad Sci USA 2006, 103(7):2257-61.

51. Kulshreshtha $\mathrm{R}$, et al: Regulation of microRNA expression: the hypoxic component. Cell Cycle 2007, 6(12):1426-31

52. Kulshreshtha $\mathrm{R}$, et al: A microRNA signature of hypoxia. Mol Cell Biol 2007, 27(5):1859-67.

53. Hebert $C$, et al: High mobility group $A 2$ is a target for miRNA-98 in head and neck squamous cell carcinoma. Mol Cancer 2007, 6:5.

54. Ho AS, et al: Circulating miR-210 as a Novel Hypoxia Marker in Pancreatic Cancer. Transl Oncol 2010, 3(2):109-13.

55. Camps C, et al: hsa-miR-210 Is induced by hypoxia and is an independent prognostic factor in breast cancer. Clin Cancer Res 2008, 14(5):1340-8.

56. Puissegur MP, et al: miR-210 is overexpressed in late stages of lung cancer and mediates mitochondrial alterations associated with modulation of HIF-1 activity. Cell Death Differ 2010.

57. Neal CS, et al: The VHL-dependent regulation of microRNAs in renal cancer. BMC Med 2010, 8:64.

58. Hua Z, et al: MiRNA-directed regulation of VEGF and other angiogenic factors under hypoxia. PLoS One 2006, 1:e116.

59. Chan JA, Krichevsky AM, Kosik KS: MicroRNA-21 is an antiapoptotic factor in human glioblastoma cells. Cancer Res 2005, 65(14):6029-33.

60. Alleman WG, et al: The in vitro and in vivo effects of re-expressing methylated von Hippel-Lindau tumor suppressor gene in clear cell renal carcinoma with 5-aza-2'-deoxycytidine. Clin Cancer Res 2004 10(20):7011-21.

61. Abe T, et al: Upregulation of BNIP3 by 5-aza-2'-deoxycytidine sensitizes pancreatic cancer cells to hypoxia-mediated cell death. Journal of gastroenterology 2005, 40(5):504-10.

62. Kim HJ, Bae SC: Histone deacetylase inhibitors: molecular mechanisms of action and clinical trials as anti-cancer drugs. Am J Transl Res 3(2):166-79.

63. Atadja PW: HDAC inhibitors and cancer therapy. Prog Drug Res 2011, 67:175-95.

64. Venugopal B, Ea TR: Developing Histone Deacetylase Inhibitors as AntiCancer Therapeutics. Curr Med Chem 2011, 18(11):1658-71.

65. Chen S, Sn N: Histone deacetylase inhibitors: the epigenetic therapeutics that repress hypoxia-inducible factors. J Biomed Biotechnol 2011, 1979-46. 
66. Fath DM, et al: Histone deacetylase inhibitors repress the transactivation potential of hypoxia-inducible factors independently of direct acetylation of HIF-alpha. J Biol Chem 2006, 281(19):13612-9.

67. Dioum EM, et al: Regulation of hypoxia-inducible factor 2alpha signaling by the stress-responsive deacetylase sirtuin 1. Science 2009, 324(5932):1289-93.

68. Kim SH, Kim KW, Jeong JW: Inhibition of hypoxia-induced angiogenesis by sodium butyrate, a histone deacetylase inhibitor, through hypoxiainducible factor-1alpha suppression. Oncol Rep 2007, 17(4):793-7.

69. Verheul HM, et al: Combination strategy targeting the hypoxia inducible factor-1 alpha with mammalian target of rapamycin and histone deacetylase inhibitors. Clin Cancer Res 2008, 14(11):3589-97.

70. Weiler J, Hunziker J, HI J: Anti-miRNA oligonucleotides (AMOs): ammunition to target miRNAs implicated in human disease? Gene Ther 2006, 13(6):496-502.

71. Orom UA, Kauppinen S, Ln AH: LNA-modified oligonucleotides mediate specific inhibition of microRNA function. Gene 2006, 372:137-41.

72. Krutzfeldt J, et al: Specificity, duplex degradation and subcellular localization of antagomirs. Nucleic Acids Res 2007, 35(9):2885-92.

73. Rushworth SA: Targeting the oncogenic role of miRNA in human cancer using naturally occurring compounds. British journal of pharmacology 2011, 162(2):346-8.

74. Li LN, et al: Down-regulation of some miRNAs by degrading their precursors contributes to anti-cancer effect of mistletoe lectin-I. British journal of pharmacology 2011, 162(2):349-64.

75. Chintala S, et al: Se-methylselenocysteine sensitizes hypoxic tumor cells to irinotecan by targeting hypoxia-inducible factor 1alpha. Cancer chemotherapy and pharmacology 2010, 66(5):899-911.

76. Gao P, et al: HIF-dependent antitumorigenic effect of antioxidants in vivo. Cancer Cell 2007, 12(3):230-8.

77. Devlin C, et al: miR-210: More than a silent player in hypoxia. IUBMB Life 2011, 63(2):94-100.

78. Wang J, et al: MicroRNAs in plasma of pancreatic ductal adenocarcinoma patients as novel blood-based biomarkers of disease. Cancer Prev Res (Phila) 2009, 2(9):807-13.

79. Gee HE, et al: hsa-mir-210 is a marker of tumor hypoxia and a prognostic factor in head and neck cancer. Cancer 2010, 116(9):2148-58.

80. Beck S: Taking the measure of the methylome. Nat Biotechnol 2010, 28(10):1026-8.

doi:10.1186/1868-7083-3-9

Cite this article as: Thirlwell et al:: Suffocating cancer: hypoxiaassociated epimutations as targets for cancer therapy. Clinical Epigenetics 2011 3:9.

\section{Submit your next manuscript to BioMed Central and take full advantage of:}

- Convenient online submission

- Thorough peer review

- No space constraints or color figure charges

- Immediate publication on acceptance

- Inclusion in PubMed, CAS, Scopus and Google Scholar

- Research which is freely available for redistribution 